\title{
NIVELES DE ANTICUERPOS BACTERICIDAS FRENTE A MENINGOCOCO C TRAS LA VACUNACIÓN DE NIÑOS DE 2 A 6 AÑOS DE EDAD EN ANDALUCÍA
}

\author{
Elena Delgado Torralbo (1,3), Julio A. Vazquez Moreno(2), Javier García León(3), Jesús González \\ Enríquez (4), Ferrán Martínez Navarro (1,5), Sonsoles Berrón Morato (2), José María Mayoral \\ Cortés (6), Mª Angeles Rubin Gómez (7), Camila Méndez Martínez (8), Margarita Cortés Majó (8), \\ Mónica Chaves Caballero (9), M $M^{\mathrm{a}}$ Luisa Bernal González (3).
}

(1) Programa de Epidemiología Aplicada de Campo (PEAC). Instituto de Salud "Carlos III"

(2) Centro Nacional de Microbiología - Instituto de Salud "Carlos III"

(3) Dirección General de Salud Pública y Participación - Consejería de Salud de la Junta de Andalucía.

(4) Agencia de Evaluación de Tecnologías Sanitarias - Instituto de Salud Carlos III.

(5) Centro Nacional de Epidemiología - Instituto de Salud Carlos III.

(6) Coordinación Epidemiología y Programas. Distrito Sanitario de Camas. Sevilla.

(7) Coordinación Epidemiología y Programas. Distrito Sanitario de Baza. Granada.

(8) Coordinación Epidemiología y Programas. Distrito Sanitario Alcalá de Guadaira-Dos Hermanas-Utrera-Morón. Sevilla.

(9) Servicio de Microbiología. Hospital Universitario Ntra. Sra. De Valme. Sevilla.

\section{RESUMEN}

Fundamento: En 1997 el 18,5\% de los casos de Enfermedad Meningocócica por serogrupo $C$ en Andalucía fueron niños de 2 a 4 años de edad; edades donde respuesta inmune inicial y duración de la vacuna antimeningocócica de polisacárido capsular $\mathrm{A}+\mathrm{C}$, es menor que en edades superiores. Se diseñó una investigación para medir la respuesta inmune producida por esta vacuna, en niños de 2 a 6 años de edad, y compararla con la inmunidad natural presente en niños no vacunados.

Métodos: 1. Doble estudio de seguimiento: i) grupos de niños previamente vacunados y grupos control, b) grupo de niños que iba a ser vacunado, para análisis pre y postvacumal $(1,6$ y 12 meses) y grupo control. II.- La actividad bactericida se midió según protocolo estandarizado del CDC frente a cepa de $N$. meningítidis $\mathrm{C}-11$. Los sueros con título de actividad bactericida (TAB) $\geq 1: 8$ se consideraron protectores.

Resultados: Al mes y a los 2 meses de vacunarse la proporción de $\mathrm{TAB} \geq 1: 8$ era significativamente superior a la del grupo control $(65.5 \%$ y $73,9 \%$ frente a $2,2 \%$ y $12 \%)$. En el prevacunal y en el postvacunal a los $6,7,12$ y 13 meses no se observó diferencia significativa entre vacunados y controles.

Conclusiones: Las diferencias entre vacunados y no vacunados 1 y 2 meses después de la vacunación indican seroconversión en los vacunados. Para el grupo de edad de 2 a 6 años la actividad bactericida adquirida declina rápidamente, ya que a los 6 meses dejan de observarse diferencias con el grupo control. gocócica.

Palabras clave: Enfermedad meningocócica. Vacuna antimenin-

Correspondencia:

Elena Delgado Torralbo

Servicio de Salud

Delegación Provincial de Sevilla

Avda. Luis Montoto, 87.

41007. Sevilla

Correo electrónico: dseedt@csalud.junta-andalucia.es
ABSTRACT

\section{Levels of Bactericidal Antibodies against Meningococcus $\mathrm{C}$ Following the Vaccination of Children between 2 and 6 years of age in Andalusia}

Background: In 1997, 18.5\% of the cases of Meningococcal Disease caused b serogroup $C$ in Andalusia were clildren between 2 and 4 years of age; ages where the initial immune response and the duration of the capsular $\mathrm{A}+\mathrm{C}$ meningococcal polyssacharide vaccine is less than in older age groups. Research was designed in orde: to measure the inmune response produced by this vaccine in children from 2 to 6 years of age and to compare it with the natural immunity present in unvaccinated children.

Methods: I. Dual monitoring study: a) groups of children vaccinated previously and control groups, b) groups of cliildren who were going to be vaccinated, for pre and post-vaccination $(1,6$ and 12 months) analysis and a control group. II. The bactericidal activity was regard to the strain of $N$. meningitidis $C-11$. The sera with bactericidal activity $(\mathrm{TAB})>1: 8$ were considered to be protective.

Results: 1 and 2 months following vaccination, the proportion of TAB $>1: 8$ was significantly higher than that of the control group of $(65.6 \%$ and $73 \%$ in comparison to $2.2 \%$ and $12 \%$ ). In the pre-vaccine and post-vaccine (after $6,7,12$ and 13 months) verification, no significant difference between vaccinated individuals and controls was observed.

Conchusions: The differences between vaccinated and unvaccinated individuals 1 and 2 months following vaccination indicate seroconversion in the vaccinated individuals. For the age group of between 2 to 6 years of age, the bactericidal activity acquired decline quickly, as, after 6 months, differences between this group and the control group are no longer observed.

Key words: Meningococcal disease. Vaccination. 


\section{INTRODUCCIÓN}

Desde el año 1994 se viene produciendo en España un cambio en el patrón epidemiológico de la enfermedad meningocócica, caracterizado por un aumento de la incidencia, un predominio del serogrupo $\mathrm{C}$, la aparición de una nueva cepa ( $\mathrm{C}: 2 \mathrm{~b}: \mathrm{P} 1.2,5)$, cierta desviación de la incidencia hacia edades superiores e incremento de la letalidad ${ }^{1-3}$. Esta nueva situación condujo a la valoración de la utilización de la vacuna antimeningocócica de polisacárido capsular $\mathrm{A}+\mathrm{C}$ cn el grupo de edad de 18 meses a 19 años y, finalmente, a su utilización en amplias zonas del país ${ }^{4}$.

En Andalucía, en el período comprendido cntre el 3 de febrero y el 7 de marzo de 1997 , se produjeron 5 agrupamientos de casos por Neisseria meningítidis $\mathrm{C}$, en los que se indicó la vacunación de la población diana (de 18 meses a 18 años de edad) por cumplirse los criterios poblacionales y temporo-espaciales establecidos. Esta situación constituía un hecho sin precedentes en la historia de la enfermedad meningocócica en Andalucía, a excepción de un agrupamiento de cuatro casos en Benamaurel (Granada) en el año 1996.

En 1.997, el 18,5\% de los casos de enfermedad meningocócica por serogrupo $\mathrm{C}$ (EMC) ocurridos en Andalucía se produjeron en niños de 2 a 4 años. En estas edades la respuesta inmune inicial y la duración de la vacuna antimeningocócica de polisacárido capsular es menor que en edades superiores ${ }^{5-12}$. Las características de esta respuesta y la eficacia y efectividad de la vacuna en estas edades no ha sido suficientemente precisada por los estudios disponibles ${ }^{13,14}$ y persiste la incertidumbre sobre los efectos de la vacuna, debido a la variabilidad e inconsistencia entre los estudios.

La mayoría de los estudios publicados sobre la capacidad inmunogénica de la vacuna miden anticuerpos totales anti-polisacárido capsular del serogrupo $\mathrm{C}$, antes y después de la vacunación. Muy pocos estudios han medido la actividad bactericida del suero de niños vacunados menores de 4 años $^{9-11}$ y generalmente son estudios no controlados.
Con el objeto de medir la respuesta inmune y su duración en niños de 2 a 4 años en nuestro ámbito, se procedió a investigar los niveles de anticuerpos bactericidas que presentaban los niños a los 2 meses de recibir vacuna antimeningocócica $\mathrm{A}+\mathrm{C}$, y los niños a los 13 meses de recibir dicha vacuna, comparando los niveles alcanzados con los observados en dos grupos de niños controles no vacunados (Estudio I). Así mismo y con motivo de la ocurrencia de un nuevo agrupamiento de casos, se investigó la seroconversión producida por la vacuna en niños de 2 a 6 años de edad, midiendo los niveles de anticuerpos bactericidas que presentaban niños previamente a la vacunación, así como al mes, a los 6 y a los 12 meses después de haberla recibido, comparando igualmente los niveles alcanzados con los observados en un grupo de niños control no vacunados (Estudio II).

\section{MATERIAL Y METODO}

\section{Población de estudio}

- Estudio I

Los sujetos incluidos en el estudio eran niños de 2 a 4 años, seleccionados de los listados del Programa de Vacunas de Andalucía por muestreo aleatorio estratificado por edad. La sustitución, por negativa a participar o por ausencia o exclusión del seleccionado, siguió el criterio de edad o proximidad de la fecha de nacimiento. Se seleccionaron 48 niños con experiencia previa de vacunación antimeningocócica y 48 niños sin experiencia previa de vacunación, que sirvieron como controles. Se estudiaron dos grupos de niños vacunados con sus respectivos controles:

- Un grupo de 23 niños vacunados 2 meses antes del inicio del estudio. Eran niños residentes en Burguillos (Sevilla), localidad de 3.400 habitantes, donde se había vacunado a la población diana tras la aparición de dos casos de EMC en un periodo de cuatro semanas.

- Un grupo de 25 niños no vacunados de Castilblanco de los Arroyos (Sevilla), 


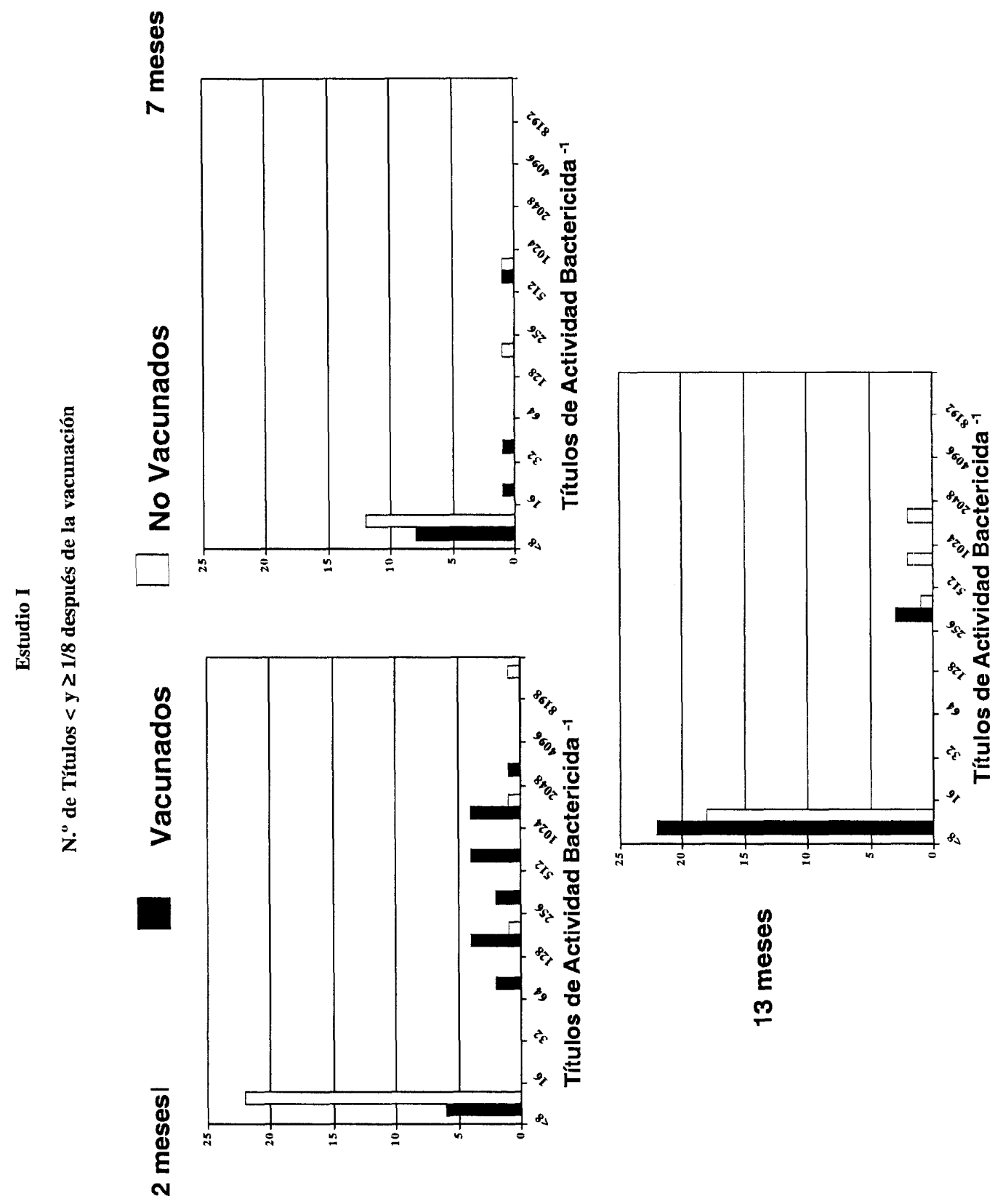


N..$^{\circ}$ de Títulos $<y \geq 1 / 8$ antes y después de la vacunación
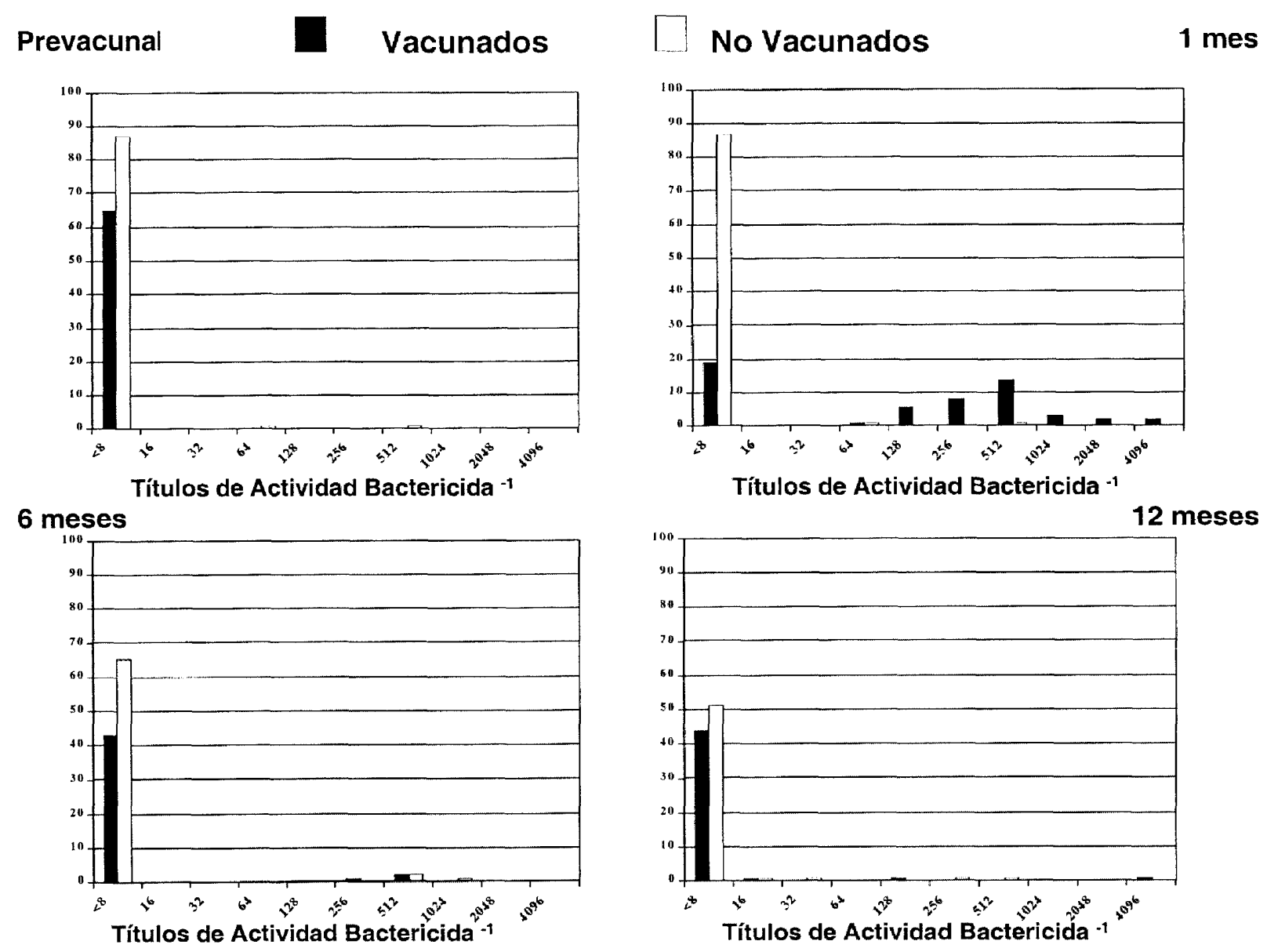
localidad de 4.045 habitantes, fue utilizado como grupo control.

- Un grupo de 25 niños vacunados 13 meses antes del inicio del estudio. Eran niños residentes en Benamaurel (Granada), localidad de 2.561 habitantes, en la que se había producido en 1.996 un agrupamiento de cuatro casos de EMC en el periodo de 8 semanas que había obligado a vacunar a la población diana.

- Un grupo de 23 niños no vacunados de Cúllar - Baza (Granada), localidad de 5.475 habitantes, fue utilizado como grupo control.

Con posterioridad, 11 niños del grupo de Burguillos y 14 del de Castilblanco fueron analizados de nuevo a los 7 meses de la fecha de la vacunación en Burguillos.

\section{- Estudio II}

Los sujetos incluidos eran niños de 2 a 6 años, seleccionando los que iban a ser vacunados de la única guardería municipal, de un colegio público y de la consulta de pediatría a demanda y los controles de la consulta de pediatría a demanda. La sustitución, por negativa a participar o por ausencia o exclusión del sujeto seleccionado, siguió el criterio de edad o proximidad de la fecha de nacimiento. Se estudió un grupo de niños que iban a ser vacunados, antes y después de recibir la vacunación con sus respectivos controles:

\section{Prevacunal:}

- Un grupo de 65 niños que iban a ser vacunados. Eran niños residentes en Utrera (Sevilla), localidad de 46.173 habitantes, donde se iba a proceder a la vacunación tras la aparición de cinco casos de EMC en un periodo de once semanas.

- Un grupo de 89 niños residentes en Arahal (Sevilla) y Las Cabezas (Sevilla), localidades de 18.375 y 15.930 habitantes, respectivamente, como grupo control.

\section{Postvacunal al mes:}

- Un grupo de 55 niños de entre los anteriores, al mes de la vacunación.

- Los controles no fueron analizados al mes por estimarse de escaso interés el cambio que pudiera haberse producido y dada la corta edad de los participantes.

\section{Postvacunal a los 6 meses:}

- Un grupo de 46 niños de entre los anteriores, a los 6 meses de la vacunación.

- Un grupo de 68 niños de entre los anteriores controles.

\section{Postvacunal a los 12 meses:}

- Un grupo de 47 niños de entre los anteriores, a los 12 meses de la vacunación.

- Un grupo de 55 niños de entre los anteriores controles.

En ambos estudios las localidades a las que pertenecían los grupos controles eran próximas y sociodemográficamente similares a las de los vacunados. En ellas también había circulado la Neisseria meningítidis $\mathrm{C}$ por las mismas fechas, produciéndose casos de enfermedad, pero no agrupamientos que indicaran la vacunación.

Se establecieron como motivos de exclusión la existencia de condiciones o patologías que afectan la respuesta inmune: 1) inmunodeficiencias congénitas o adquiridas, incluídas las de causa yatrogénica; 2 ) haber padecido previamente enfermedad meningocócica. También fue motivo de exclusión encontrarse bajo tratamiento antibiótico en los 10 días previos al estudio. En total por este último motivo resultaron excluidos 5 niños en el Estudio I.

\section{Cuestionario:}

Se solicitó a los padres de los niños que contestaran un cuestionario que incluía datos de filiación, características personales (edad y 
género), sociodemográficas (escolaridad, tamaño de la vivienda y número de convivientes), antecedentes de contacto con caso de enfermedad meningocócica y motivos de exclusión. Igualmente, se solicitaba la aceptación de participación en el estudio mediante la firma del documento de consentimiento informado.

\section{Ensayo bactericida del suero:}

Se extrajo una muestra de $10 \mathrm{ml}$ de sangre a los niños, la cual fue analizada por el Laboratorio de Referencia de Neisserias del Centro Nacional de Microbiología. En el estudio se determinó el nivel de anticuerpos bactericidas, o actividad bactericida, mediante el Ensayo Bactericida del suero, considerando como nivel protector un título de anticuerpos bactericidas (TAB) igual o mayor a 1:8.

Esta técnica se realizó según los protocolos estandarizados del Center for Disease Control and Prevention (CDC) de Atlanta. Para medir la actividad bactericida los sueros son previamente inactivados a $56^{\circ} \mathrm{C}$ durante 30 minutos. Como fuente de complemento se utiliza suero estéril de conejos de menos de cuatro semanas, debido a que éste presenta baja toxicidad para la cepa C11, que es la utilizada. Los sueros, en presencia de complemento, son enfrentados en diluciones dobles, desde $1: 8$ a 1:512, a la cepa C11. A las 18 horas se cuentan las bacterias (colonias) supervivientes y los títulos son definidos como aquella dilución capaz de matar el 50\% o más del inóculo bacteriano, comparado con el crecimiento de la cepa a tiempo 0. Como control se utilizaron sueros de referencia suministrados por el CDC, en los que previamente había sido establecida la actividad bactericida.

\section{Análisis:}

Se analizaron:

- Las diferencias por género, edad, escolaridad y número de convivientes entre los grupos de vacunados y los de sus controles (test de Ji cuadrado y Exacto de Fisher).
- Las diferencias entre las proporciones de $T A B$ igual o mayor a 1:8 en niños vacunados y en controles (test de Ji cuadrado y Exacto de Fisher).

- Las diferencias entre las proporciones de $\mathrm{TAB}$ igual o mayor a 1:8 en niños vacunados de cada año de edad (test de Ji cuadrado y Exacto de Fisher).

- Las diferencias entre las medianas de los $\mathrm{TAB}$ alcanzados en los vacunados y en los controles (test de MannWhitney).

- Las diferencias entre las medianas de los $\mathrm{TAB}$ alcanzados en niños vacunados de cada año de edad (test de MannWhitney y Anova no paramétrico).

- Las diferencias entre la primera y segunda medición realizada en niños de Burguillos y Castilblanco de los Arroyos (test de McNemar y test de Wilcoxon).

- Las diferencias entre las mediciones realizadas en niños de Utrera y Arahal y Las Cabezas en los distintos momentos (test de McNemar y test de Wilcoxon).

Fueron utilizados los programas informáticos Epiinfo Versión 6.0 y Statgraphics. Plus.

\section{RESULTADOS}

Población de estudio: No se observaron diferencias estadísticamente significativas en la distribución de las variables género, edad, escolarización y número de convivientes entre los grupos de vacunados y sus controles (tablas 1 y 2 ).

\section{Actividad bactericida frente al meningococo serogrupo $C$ :}

\section{Estudio I}

Se observó que la proporción de niños que presentaban TAB igual o mayor a 1:8 en el grupo de vacunados 2 meses antes era significativamente superior a la de su grupo control 
Tabla 1

Estudio I. Comparabilidad de los grupos de vacunados y de control. Andalucía 1997

\begin{tabular}{|c|c|c|c|c|c|c|}
\hline & \multicolumn{3}{|c|}{ Burguillos-Castilblanco de los Arroyos } & \multicolumn{3}{|c|}{ Benamaurel-CúllarBaza } \\
\hline & $\begin{array}{l}\text { Vacunados } \\
\text { (I) }\end{array}$ & $\begin{array}{c}\text { No vacunados } \\
\text { (2) }\end{array}$ & $\begin{array}{c}\text { Comparación } \\
\text { (1)-(2)* }\end{array}$ & $\begin{array}{l}\text { Vacunados } \\
\text { (3) }\end{array}$ & $\begin{array}{c}\text { No vacunados } \\
\text { (4) }\end{array}$ & $\begin{array}{c}\text { Comparación } \\
\text { (3)-(4)* }\end{array}$ \\
\hline $\begin{array}{l}\text { Género } \\
\text { Masculino } \\
\text { Femenino }\end{array}$ & $\begin{array}{c}9 \\
14\end{array}$ & $\begin{array}{l}13 \\
12\end{array}$ & $\begin{array}{l}\text { NS } \\
\text { NS }\end{array}$ & $\begin{array}{l}14 \\
11\end{array}$ & $\begin{array}{l}12 \\
11\end{array}$ & $\begin{array}{l}\text { NS } \\
\text { NS }\end{array}$ \\
\hline $\begin{array}{l}\text { Edad } \\
\text { 2años } \\
\text { 3años } \\
\text { 4años }\end{array}$ & $\begin{array}{c}5 \\
8 \\
10\end{array}$ & $\begin{array}{l}7 \\
11 \\
7\end{array}$ & $\begin{array}{l}\text { NS } \\
\text { NS } \\
\text { NS }\end{array}$ & $\begin{array}{l}8 \\
11 \\
6\end{array}$ & $\begin{array}{l}8 \\
8 \\
7\end{array}$ & $\begin{array}{l}\text { NS } \\
\text { NS } \\
\text { NS }\end{array}$ \\
\hline $\begin{array}{l}\text { Escolarización } \\
\mathrm{Si} \\
\text { No }\end{array}$ & $\begin{array}{l}17 \\
6\end{array}$ & $\begin{array}{l}22 \\
3\end{array}$ & $\begin{array}{l}\text { NS } \\
\text { NS }\end{array}$ & $\begin{array}{l}16 \\
6\end{array}$ & $\begin{array}{l}15 \\
7\end{array}$ & $\begin{array}{l}\text { NS } \\
\text { NS }\end{array}$ \\
\hline $\begin{array}{l}\text { Convivientes } \\
3 \\
4 \\
5 \\
6 \text { ó más }\end{array}$ & $\begin{array}{l}5 \\
10 \\
6 \\
2\end{array}$ & $\begin{array}{l}2 \\
9 \\
7 \\
7\end{array}$ & $\begin{array}{l}\text { NS } \\
\text { NS } \\
\text { NS } \\
\text { NS }\end{array}$ & $\begin{array}{l}6 \\
11 \\
5 \\
3\end{array}$ & $\begin{array}{c}2 \\
13 \\
2 \\
4\end{array}$ & $\begin{array}{l}\text { NS } \\
\text { NS } \\
\text { NS } \\
\text { NS }\end{array}$ \\
\hline
\end{tabular}

* test de ji cuadrado, test Exacto de Fisher

NS: diferencia no significativa

(73,9\% frente a $12,0 \%)$. También se observó diferencia entre la mediana de los TAB alcanzados por cada grupo $(1: 128$ frente a $<1: 8)$ (tabla 3).

Entre los niños vacunados no se observaron diferencias en la Actividad Bactericida (proporción de $\mathrm{TAB}$ protectores y medianas de los TAB alcanzados) según la edad.

A los 7 meses de la vacunación los resultados no mostraron diferencias significativas en la proporción de TAB protectores entre el grupo de vacunados y el de sus controles (18,2 frente a 14,3). Tampoco se observaron diferen- cias en la mediana de los TAB alcanzados por cada grupo (<1:8 en ambos) (tabla 3 ).

Entre los niños vacunados, tanto la probabilidad de tener $\mathrm{TAB}$ protectores como la mediana de los TAB alcanzados, es significativamente superior a los 2 meses de la vacunación que a los 7 meses. En el grupo control no se observaron estas diferencias. (tabla 3 ).

No se observaron diferencias significativas entre el grupo de vacunados 13 meses antes y el de sus controles, ni en la proporción de TAB protectores $(12,0$ frente a 21,7$)$ ni en la mediana de los TAB alcanzados $(<1: 8$ en ambos grupos) (tabla 3). 
Tabla 2

Estudio II. Comparabilidad de los grupos de vacunados y de control. Andalucía 1997

\begin{tabular}{|l|c|c|c|}
\hline \multirow{2}{*}{} & \multicolumn{3}{|c|}{ Utrera-Arahal y Las Cabezas } \\
\cline { 2 - 4 } & $\begin{array}{c}\text { Vacunados } \\
(I)\end{array}$ & $\begin{array}{c}\text { No vacunados } \\
(2)\end{array}$ & $\begin{array}{c}\text { Comparación } \\
(1)-(2)^{*}\end{array}$ \\
\hline Género & & & \\
Masculino & 30 & 45 & NS \\
Femenino & 35 & 44 & NS \\
\hline Edad & & & \\
2 años & 10 & 15 & NS \\
3 años & 12 & 23 & NS \\
4 años & 19 & 19 & NS \\
5 años & 20 & 17 & NS \\
6 años & 4 & 15 & NS \\
\hline Escolarización & 52 & 69 & NS \\
Sí & 9 & 18 & NS \\
No & 8 & 15 & NS \\
\hline Convivientes & & 32 & NS \\
3 & 15 & 20 & NS \\
4 & 12 & 20 & NS \\
\hline 5 & 6 ó más &
\end{tabular}

* test de ji cuadrado, test Exacto de Fisher. NS: diferencia no significativa.

\section{Estudio II}

En el momento prevacunal no se observó diferencia estadísticamente significativa entre la proporción de TAB igual o mayor de $1: 8 \mathrm{de}$ los niños que iban a ser vacunados y la de su grupo control $(0,0 \%$ frente a $2,2 \%)$. Tampoco se observó entre la mediana de los TAB alcanzados por cada grupo (1:8 en ambos) (tabla 4 ).

Al mes de la vacunación se observó que la proporción de niños vacunados que presentaban TAB igual o mayor de 1:8 era significativamente superior a la de su grupo control $(65,5 \%$ frente a $2,2 \%)$. Se observó también diferencia entre la mediana de los TAB alcanzados por cada grupo $(1 / 256$ frente $a<1: 8)$ (tabla 4).

A los 6 y a los 12 meses de la vacunación los resultados no mostraron diferencias significativas en la proporción de TAB protectores entre el grupo de vacunados y el de sus controles $(6,5 \%$ y $6,4 \%$ frente a $4,4 \%$ y $7,3 \%) \mathrm{ni}$ en la mediana de los TAB alcanzados por cada grupo ( $<1: 8$ y $<1: 8$ en ambos) (tabla 4).

Entre los vacunados, a diferencia de lo observado entre los controles, tanto la probabi-

Tabla 3

Estudio $\mathrm{I}$. Niveles de anticuerpos bactericidas frente al meningococo $\mathrm{C}$ en grupos de niños vacunados con vacuna antimeningocócica de polisacárido capsular $(a+c)$ y en grupos control. Andalucía 1997

\begin{tabular}{|c|c|c|c|c|c|c|c|c|c|c|c|c|}
\hline \multirow{3}{*}{$\begin{array}{l}\text { Tiempo } \\
\text { respecto a la } \\
\text { vacunación }\end{array}$} & \multicolumn{5}{|c|}{ Vacunados } & \multicolumn{5}{|c|}{ No vacunados } & \multicolumn{2}{|c|}{ Comparación } \\
\hline & \multirow{2}{*}{$\begin{array}{l}N . " d e \\
\text { niños }\end{array}$} & \multicolumn{2}{|c|}{ Título $\geq 1 / 18$} & \multirow{2}{*}{$\begin{array}{l}\text { IC } 95 \% \\
\text { del }(\%)\end{array}$} & \multirow{2}{*}{$\begin{array}{c}\text { Título } \\
\text { Mediana } \\
\left(\text { Rango }{ }^{-1}\right)\end{array}$} & \multirow{2}{*}{$\begin{array}{l}N .^{0} d e \\
\text { niños }\end{array}$} & \multicolumn{2}{|c|}{ Título $\geq 1 / 18$} & \multirow{2}{*}{$\begin{array}{l}I C 95 \% \\
\operatorname{del}(\%)\end{array}$} & \multirow{2}{*}{$\begin{array}{c}\text { Título } \\
\text { Mediana } \\
\left(\text { Rango }^{-1}\right)\end{array}$} & \multirow{2}{*}{$\% p^{I}$} & \multirow{2}{*}{ Mediana $p^{2}$} \\
\hline & & $N$ & $\%$ & & & & $N$ & $\%$ & & & & \\
\hline & \multicolumn{12}{|c|}{ Burguillos - Castilblanco de los Arroyos } \\
\hline 2 meses & 23 & 17 & 73,9 & $51,6-89,8$ & $\begin{array}{c}1 / 128 \\
(1-2048)\end{array}$ & 25 & 3 & 12,0 & $2,5-31,2$ & $\begin{array}{c}<1 / 8 \\
(1-8192)\end{array}$ & 0,0001 & 0,0001 \\
\hline \multirow[t]{2}{*}{7 meses } & 11 & 3 & $18,2^{*}$ & $2,3-51,8$ & $\begin{array}{l}<1 / 8^{* *} \\
(1-512)\end{array}$ & 14 & 2 & 14,3 & $1,8-42,8$ & $\begin{array}{c}<1 / 8 \\
(1-512)\end{array}$ & $\mathrm{N} / \mathrm{S}$ & $\mathrm{N} / \mathrm{S}$ \\
\hline & \multicolumn{12}{|c|}{ Benamaurel-Cúllar Baza } \\
\hline 13 meses & 25 & 3 & 12,0 & $2,5-31,2$ & $\begin{array}{c}<1,8 \\
(1-256)\end{array}$ & 23 & 5 & 21,7 & $7,4-43,7$ & $\begin{array}{c}<1 / 8 \\
(1-1024)\end{array}$ & $\mathrm{N} / \mathrm{S}$ & $\mathrm{N} / \mathrm{S}$ \\
\hline
\end{tabular}

\footnotetext{
$\mathrm{p}^{1}$ Fisher. $\quad *$ Diferencia significativa respecto al Tiempo anterior (Mc Nemar)

NS: diferencia no significativa.

$\mathrm{p}^{2}$ Mann-Whitney. $\quad * *$ Diferencia significativa respecto al Tiempo anterior (Wilcoxon).
} 
Tabla 4

Estudio II. Niveles de anticuerpos bactericidas frente al meningococo $\mathrm{C}$ en grupos de niños vacunados con vacuna antimeningocócica de polisacárido capsular (a+c) y en grupos control. Andalucía 1997

\begin{tabular}{|c|c|c|c|c|c|c|c|c|c|c|c|c|}
\hline \multirow{3}{*}{$\begin{array}{c}\text { Tiempo } \\
\text { respecto a la } \\
\text { vacunación }\end{array}$} & \multicolumn{5}{|c|}{ Vacunados } & \multicolumn{5}{|c|}{ No vacunados } & \multicolumn{2}{|c|}{ Compararión } \\
\hline & \multirow{2}{*}{$\begin{array}{l}N .^{o} d e \\
\text { niños }\end{array}$} & \multicolumn{2}{|c|}{ Título $\geq 1 / 18$} & \multirow{2}{*}{$\begin{array}{l}\text { IC } 95 \% \\
\operatorname{del}(\%)\end{array}$} & \multirow{2}{*}{$\begin{array}{c}\text { Título } \\
\text { Mediana } \\
\left(\text { Rango }^{-1}\right)\end{array}$} & \multirow{2}{*}{$\begin{array}{l}N .^{\circ} \text { de } \\
\text { niños }\end{array}$} & \multicolumn{2}{|c|}{ Título $\geq 1 / 18$} & \multirow{2}{*}{$\begin{array}{l}I C 95 \% \\
\operatorname{del}(\%)\end{array}$} & \multirow{2}{*}{$\begin{array}{c}\text { Título } \\
\text { Mediana } \\
(\text { Rango-1) }\end{array}$} & \multirow{2}{*}{$\% p^{l}$} & \multirow{2}{*}{ Mediana $p^{2}$} \\
\hline & & $N$ & $\%$ & & & & $N$ & $\%$ & & & & \\
\hline \multicolumn{13}{|c|}{ Utrera-Arahal / Las Cabezas } \\
\hline Prevacunal & 65 & 0 & 0,0 & $0,0-5,5$ & $\begin{array}{c}<1 / 8 \\
(-)\end{array}$ & 89 & 2 & 2,2 & $0,3-7,9$ & $\begin{array}{c}<1 / 8 \\
(1-512)\end{array}$ & $\mathrm{N} / \mathrm{S}$ & $\mathrm{N} / \mathrm{S}$ \\
\hline 1 mes & 55 & 36 & $65,5 *$ & $51,5-77,8$ & $\begin{array}{l}1 / 256^{* * *} \\
(1-4096)\end{array}$ & 89 & 2 & 2,2 & $0,3-7,9$ & $\begin{array}{c}<1 / 8 \\
(1-512)\end{array}$ & 0,0001 & 0,0001 \\
\hline 6 meses & 46 & 3 & $6,5^{*}$ & $1,4-17,9$ & $\begin{array}{l}<1 / 8^{* * *} \\
(1-512)\end{array}$ & 68 & 3 & 4,4 & $0,9-2,4$ & $\begin{array}{c}<1 / 8 \\
(1-1024)\end{array}$ & $\mathrm{N} / \mathrm{S}$ & $N / S$ \\
\hline 12 meses & 47 & 3 & $6,4^{* *}$ & $1,3-17,5$ & $\begin{array}{l}<1 / 8 * * * * \\
(1-4096)\end{array}$ & 55 & 4 & 7,3 & $2,0-7,6$ & $\begin{array}{c}<1 / 8 \\
(1-512)\end{array}$ & $\mathrm{N} / \mathrm{S}$ & $\mathrm{N} / \mathrm{S}$ \\
\hline
\end{tabular}

$p^{i} \quad$ Fisher

$\mathrm{p}^{2} \quad$ Mann-Whitney.

* Diferencia significativa respecto al Tiempo anterior (Mc Nemar).

** Diferencia significativa respecto a 1 mes (Mc Nemar).

*** Diferencia significativa respecto al Tiempo anterior (Wilcoxon).

**** Diferencia significativa respecto a 1 mes (Wilcoxon).

NS: Diferencia no significativa.

lidad de tener TAB protectores como la mediana de los TAB alcanzados, es significativamente superior al mes de la vacunación que en el momento prevacunal y que a los 6 y a los 12 meses de haberse vacunado (tabla 4).

En este estudio tampoco se observaron, entre los niños vacunados, diferencias en la Actividad Bactericida (proporción de TAB protectores y medianas de los TAB alcanzados) según la edad.

\section{DISCUSIÓN}

En los países del hemisferio norte la vacuna contra el meningococo $\mathrm{C}$ ha tenido un uso limitado, a pesar de no tratarse de una vacuna reciente. En los últimos años las experiencias habidas en diferentes lugares con la EMC ha llevado a plantear la valoración de un uso más amplio de esta vacuna. No obstante, como se desprende de diferentes investigaciones, la escasa protección que confiere la vacuna en los menores de 2 años (grupo con mayor riesgo de EMC), así como la limitada eficacia en menores de 5 años y la corta duración de la inmunidad que ofrece en estas edades, han llevado a la recomendación de usar la vacuna fundamentalmente en el control de brotes epidémicos causados por los serogrupos A y $\mathrm{C}$ y no de manera rutinaria ${ }^{15}$.

A pesar de ello, la incertidumbre en torno a la acción de la vacuna en esas edades persiste. Los estudios publicados sobre el efecto de la vacuna no suelen ser controlados. La variabilidad e inconsistencia entre los estudios no permiten precisar suficientemente las características de la respuesta inmune y la eficacia y efectividad de la vacuna en estas edades ${ }^{13,14}$. Por este motivo y ante la elevada incidencia de EMC observada en niños pequeños durante 1997 en Andalucía, se decidió realizar el presente trabajo sobre respuesta inmune en grupos de niños de 2 a 6 años de edad y su compara- 
ción con la inmunidad natural presente en grupos de niños controles no vacunados.

La mayoría de los estudios publicados sobre la capacidad inmunogénica de la vacuna miden niveles de anticuerpos totales anti-polisacárido capsular del serogrupo C (TAT). En algunos de estos trabajos se ha medido también actividad bactericida (TAB), que constituye sólo una parte de los $\mathrm{T} \Lambda \mathrm{T}^{10}$, pero son pocos los estudios que han medido actividad bactericida (TAB) en niños vacunados menores de 7 años ${ }^{9-11}$. Las discrepancias observadas entre ambas mediciones ${ }^{11}$ y el hecho de que la técnica para medir TAT, además de consumir más tiempo, presente mayor variabilidad y dificultad de interpretación, llevaron a optar por la medición de TAB en este trabajo.

Es preciso comentar que la no disponibilidad de los TAB prevacunales en el Estudio I limita atribuir al efecto de la vacuna la variación observada. No obstante, asumimos la posibilidad de valorar dicho estudio en términos de seroconversión vacunal. Para ello nos basamos tanto en las diferencias observadas entre grupos de vacunados y grupos control (grupos que comparten, junto a otras, la característica de tener experiencia frente al meningococo C) a los 2 meses y las similitudes a los 7 y a los 13 meses, como en los resultados sobre respuesta vacunal observados en estudios antes-después por otros autores ${ }^{9-11}$, así como en nuestra propia experiencia aportada por el Estudio II.

La poca edad de los participantes en estudios que conllevaron obtenciones repetidas de muestras de sangre, la no devolución de resultados de los análisis de suero a los familiares de los niños participantes y las circunstancias de alerta social presentes, especialmente en el momento prevacunal del Estudio II, fueron aspectos que dificultaron la realización del trabajo.

Los resultados obtenidos indican que el $73,9 \%$ de los niños del Estudio I y el $65,5 \%$ de los del Estudio II, mantenían TAB protectores a los 2 meses y al mes, respectivamente, de haberse vacunado. Esto constituye una respuesta inmune similar, o inclusive superior, a la observada en niños de estas edades, e infe- rior a la observada en niños mayores en estudios de seroconversión realizados por otros autores ${ }^{10}$.

A los 6 meses de la vacunación, el nivel de anticuerpos cae hasta el punto de desaparecer las diferencias entre los vacunados y sus controles, no existiendo por tanto, en nuestra experiencia, diferencia entre estar vacunado $(6,5 \%)$ y no estarlo $(4,4 \%)$ a partir de ese momento. Estos resultados vienen a indicar la limitada duración de los TAB protectores en estas edades. La Agencia de Evaluación de Tecnologías Sanitarias (AETS) ${ }^{15}$ señala la probabilidad de que la proporción de niños efectivamente protegidos al año de la vacunación sea baja. Nuestros resultados son similares a los que se han observado en las experiencias de British Columbia (Canadá) ${ }^{10}$ y Cantabria (España $)^{16}$, experiencias en las que fue llevada a cabo una amplia estrategia de vacunación. En la segunda de ellas los autores abogan por la existencia de otros factores inmunológicos, no bien conocidos, para explicar el buen efecto clínico-epidemiológico (medido por la caída en la tasa de ataque de la enfermedad) alcanzado un año después de la vacunación masiva de la población diana. En nuestra experiencia no apareció ningún caso de EMC en las localidades a las que pertenecían los niños vacunados a todo lo largo del año que siguió a la vacunación, localidades en las que, como sabemos, había sido vacunada la población diana, pero tampoco aparecieron casos de EMC en las localidades a las que pertenecían los grupos control y en las cuales no se había vacunado.

No obstante los resultados comentados, debemos recordar que no existe acuerdo en cuanto al nivel preciso de actividad bactericida que proporciona una eficaz protección clínica. En este trabajo se optó por utilizar como punto de corte la dilución 1:8. En el Estudio II los sueros correspondientes a los 6 meses y a los 12 meses de la vacunación fueron analizados también utilizando como punto de corte la dilución 1:4, no obteniéndose resultados diferentes. Los autores mencionados en el párrafo anterior utilizaron títulos tanto de 1:4 como de 1:8 y los resultados obtenidos fueron similares. En cualquier caso y como una limitación de 
estos estudios, los resultados deben interpretarse más como una aproximación que como una medida de auténtica protección.

Un hecho demostrado es la variabilidad de la respuesta vacunal scgún cl grupo de cdad, habiéndose observado que la respuesta inmune a la vacuna aumenta con la edad ${ }^{5-8,9-11}$. En nuestro trabajo no hemos obtenido este resultado. El reducido tamaño de la población estudiada, con ser un hecho frecuente en este tipo de estudios, constituye otra limitación del trabajo y afecta a la precisión de los resultados. Posiblemente este bajo número de niños estudiados y, muy especialmente, el estrecho rango de edad de los mismos (de 2 a 6 años), haya influido a la hora de no encontrar diferencias en la respuesta inmune por año de edad.

La inmunidad natural no parece haber jugado un importante papel en nuestra experiencia. Nuestros resultados ofrecen un bajo porcentaje de controles $(12,0 \%$ en el Estudio I y $2,2 \%$ en el Estudio II) con inmunidad natural, controles que sabemos pertenecen a localidades por las que había circulado la Neisseria Meningítidis dos meses antes y un mes antes respectivamente. Esto posiblemente haya que atribuirlo a lo ya observado en British Columbia (Canada) ${ }^{10}$ y en Galicia (España) ${ }^{17}$ acerca del carácter excepcional de portadores del microorganismo en la edad pediátrica, particularmente de serogrupo $C$. La baja prevalencia de portadores en estos grupos de edad explica también que nosotros no hayamos observado otras diferencias entre la respuesta inmune de los vacunados y la de sus controles que no puedan ser las, razonablemente, achacables a la vacunación. Si en las localidades donde se produjo agrupamiento de EMC. existió una mayor circulación de la Neissería y se supone un mayor número de portadores, la inmunidad natural debería haber potenciado a la adquirida por la vacuna ${ }^{5,18,19}$. Sin embargo, tanto a los 7 como a los 13 meses de la vacunación en el Estudio I, y tanto a los 6 como a los 12 meses de la vacunación en el Estudio II, no se observaron diferencias entre los vacunados -que sabemos pertenecían a localidades donde se produjo agrupamiento- y los controles - procedentes estos de localidades donde no lo hubo-. Es decir, a partir de los 6 meses de la vacunación la inmunidad adquirida es equiparable a la inmunidad natural observada en localidades similares con baja incidencia de EMC.

En cualquier caso, aunque la comparación entre los controles y los vacunados a los $6,7,12$ y 13 meses sugiere que inmunidad natural y adquirida ofrecen resultados similarmente bajos, la probabilidad de estar seroprotegido en los vacunados (adquirida) cae de manera significativa a partir de los 6 meses de la vacunación. Sin embargo, en los controles la inmunidad natural se mantiene, si bien era muy baja desde el principio. Hay que decir además que en nuestro estudio el TAB individual más elevado fue de 1:8192 y correspondió a un niño control. En general, los controles con TAB protectores presentaron niveles de titulación elevada.

\section{AGRADECIMIENTOS}

Este trabajo ha sido posible gracias a la colaboración desinteresada de profesionales sanitarios de los distritos de Atención Primaria de Baza (Granada), Camas (Sevilla), AlcaláDos Hermanas-Utrera-Morón (Sevilla), de los Laboratorios de Microbiología del Hospital General Básico de Baza (Granada) y Hospital Universitario Ntra. Sra. de Valme (Sevilla) y de las Delegaciones de Salud de Granada y Sevilla.

\section{BIBLIOGRAFÍA}

1. Centro Nacional de Epidemiología. Informe sobre la situación de la enfermedad meningocócica en España. Madrid: Centro Nacional de Epidemiología; 1997.

2. Vázquez JA. Infección meningocócica, informe del laboratorio de referencia de meningococos (1995). Bol Epidemiol Sem 1996; 129: 1307-27.

3. Mateo S, Cano R, García C. Changing epidemilogy of meningococcal disease in Spain, 1989-1997. Eurosurveillance 1997; 2 (10): 71-74.

4. Conferencia Nacional sobre Enfermedad Meningocócica. Comisión de Salud Pública 
del Consejo Interterritorial del Sistema Nacional de Salud. Oviedo, 16-17 de Junio; 1997.

5. Gold R, Lepow ML, Goldschneider I, Draper TF, Gotschlich EC. Kinetics of antibody production to group $\mathrm{A}$ and group $\mathrm{C}$ meningococcal polysaccharide vaccines administered during the first six years of life: prospects for routine immunization of infants and children. J Infect Dis 1979; 140 (5): 690-697.

6. Greenwod BM, Whittle HC, Bradley AK, Fayet MT, Gilles HM. The duration of the antibody response to meningococcal vaccination in an African village. Trans $\mathrm{R}$ Soc Trop Med Hyg 1980; 74 (6): 756-60.

7. Lepow ML, Goldschneider I, Gold R, Randolph M, Goldschlich EC. Persistence of antibody following immunization of children with groups $\mathrm{A}$ and $\mathrm{C}$ meningococcal polysaccharide vaccines. Pediatrics 1977 :60 (5): 673-80.

8. Parke JC, Schneerson R, Robbins JB, Schlesselman JJ. Interim report of a controlled trial of immunization with capsular polysaccarides of haemophilus influenzae type $b$ and group C Neisseria meningitidis in Mecklenburg County, North Carolina (March 1974-March 1976). J Infect Dis 1977; 136 (Suplement): S51-S56.

9. Peltola HP, Safary A, Káyhty H, Karanko V, André FE. Evaluation of two tetravalent (ACYW135) meningococcal vaccines in infants and small children: a clinical study comparing immunogenicity of O-AcetylNegative and O-Acetyl-Positive group C polysaccharides. Pediatrics 1985; 76 (1): 91-96.

10. Mitchell LA, Ochnio JJ, Glover C, Lee AY, Ho MKL, Bell A. Analysis of meningococcal serogroup C-specific antibody levels in british columbian children and adolescent. I Infect Dis 1996; 173: 1009-13.

11. King WJ, Mac Donald NE, Wells G, Huang J, Alen U, Chang F, et al. Total and functional antibody response to a quadrivalent meningo- coccal polysaccharide vaccine among children. J Pediatr 1996; 128(2): 196-202.

12. Mohammed I, Onyenmelukwe GC, Obineche EN, Gupta N, Oyeyinka GO. Control of epidemic meningococcal meningitis by mass vaccination. II. Persistence of antibody four years after vaccination. J Infect 1984; 9:197-202.

13. Taunay AE, Feldman RA, Bastos CO, Galvao PAA, Morais JS; Castro IO. Avaliaçao do efeito protetor de vacina polissacaídica antimeningocócica do grupo C, em crianças de 65 a 36 meses. Rev Inst Adolfo Lutz 1978;38:77-82.

14. De Wals P, Dionne M, Douville-Fradet $M$, Boulianne N, Drapeau J, De Serres G. Impact of a mass inmunization campaign against serogroup $\mathrm{C}$ meningococcus in the Province of Quebec, Canada. Bull World Health Organ 1996;74(4):407-11.

15. González Enríquez J, García Comas L, Alcaide Jiménez JF, Sáenz Calvo A y Conde Olasategui J. Eficacia de la vacuna meningocócica de polisacárido capsular del grupo $\mathrm{C}$. Rev Esp Salud Pública 1997; 71: 103-126.

16. Consejería de Sanidad, Consumo y Bienestar Social. Dirección General de Sanidad y Consumo. Seroprevalencia de anticuerpos bactericida frente al meningococo $\mathrm{C}$ en Cantabria diez meses después de la campaña de vacunación. Cantabria: Consejería de Sanidad, Consumo y Bienestar Social; 1998.

17. Dirección Xeral de Saude Pública, Xunta de Galicia: Portadores de N. meningitidis e incidencia de enfermidade meningocócica". Bol Epidemiol Galicia 1997; X(3).

18. Lepow ML, Beeler J, Randolph M, Samuelson JS and Hankins WA: Reactogenicity and immunogenicity of a quadrivalent combined meningococcal polysaccharide vaccine in children . J Infect Dis 1986, 154: 1033-1036.

19. Gold R, Lepow ML, Goldschneider I, Draper TF, Gotschlich EC. Clinical evaluation of group $\mathrm{A}$ and group $\mathrm{C}$ meningococcal polysaccharide vaccines in infants. J Clin Invest 1975;56: 1536-1547. 